Article

\title{
Fixed Point Results in Partial Symmetric Spaces with an Application
}

\author{
Mohammad Asim ${ }^{1, *(1)}$, A. Rauf Khan ${ }^{2}$ and Mohammad Imdad ${ }^{1}(\mathbb{C}$ \\ 1 Department of Mathematics, Aligarh Muslim University, Aligarh-202002, India; mhimdad@gmail.com \\ 2 Department of Applied sciences, University Polytechnic, Aligarh Muslim University, Aligarh-202002, India; \\ abdurraufamu@gmail.com \\ * Correspondence: mohdasim.rs@amu.ac.in
}

Received: 8 December 2018; Accepted: 15 January 2019; Published: 22 January 2019

\begin{abstract}
In this paper, we first introduce the class of partial symmetric spaces and then prove some fixed point theorems in such spaces. We use one of the our main results to examine the existence and uniqueness of a solution for a system of Fredholm integral equations. Furthermore, we introduce an analogue of the Hausdorff metric in the context of partial symmetric spaces and utilize the same to prove an analogue of the Nadler contraction principle in such spaces. Our results extend and improve many results in the existing literature. We also give some examples exhibiting the utility of our newly established results.
\end{abstract}

Keywords: partial symmetric; fixed point; contraction and weak contraction; Nadler's theorem

MSC: 47H10; 54H25

\section{Introduction}

The classical Banach contraction principle is one of the most powerful and effective results in analysis established by Banach [1], which guarantees the existence and uniqueness of fixed points in complete metric spaces. This principle has been extended and generalized in many different directions. One of these ways is to enlarge the class of spaces, such as partial metric spaces [2], metric-like spaces [3], b-metric spaces [4], rectangular metric spaces [5], cone metric spaces [6], and several others. Sometimes, one may come across situations wherein all the metric conditions are not needed (see [7-11]). Motivated by this reality, several authors established fixed point and common fixed point results in symmetric spaces (or semi-metric spaces).

A symmetric $d$ on a non-empty set $X$ is a function $d: X \times X \rightarrow \mathbb{R}_{+}$which satisfies $d(x, y)=d(y, x)$ and $d(x, y)=0$ if and only if $x=y$, for all $x, y \in X$. Unlike the metric, the symmetric is not generally continuous. Due to the absence of a triangular inequality, the uniqueness of the limit of a sequence is no longer ensured. To have a workable setting, Wilson [12] suggested several related weaker conditions to overcome the earlier mentioned difficulties, which we will adopt to our setting. Such weaker conditions will be stated in the preliminaries.

In 1969, Nadler [13] initiated the study of fixed points for multi-valued contractions using the Hausdorff metric, and extended the Banach fixed point theorem to set-valued contractive maps. The theory of multi-valued maps has applications in control theory, convex optimization, differential equations, economics, and so on.

On the other hand, Matthews [2] introduced the concept of partial metric spaces as a part of the study of denotational semantics of dataflow networks, and proved an analogue of the Banach contraction theorem, and Kannan-Ćirić and Ćirić quasi-type fixed point results. 
Combining the ideas involved in the concepts of partial metric spaces and symmetric spaces, we introduce the class of partial symmetric spaces, wherein we prove existence and uniqueness fixed point results for certain types of contractions in partial symmetric spaces. Furthermore, with a view to prove a multivalued analogue of Nadler's fixed point theorem, we adopt the idea of the Hausdorff metric in the setting of partial symmetric spaces. Finally, we use one of the our main results to examine the existence and uniqueness of a solution for a system of Fredholm integral equations.

\section{Preliminaries}

In this section, we collect some relevant definitions and examples which are needed in our subsequent discussions.

Now, we introduce the partial symmetric space as follows:

Definition 1. Let $X$ be a non-empty set. A mapping $\mathcal{P}: X \times X \rightarrow \mathbb{R}_{+}$is said to be a partial symmetric if, for all $x, y, z \in X$ :

(1P) $x=y$ if and only if $\mathcal{P}(x, x)=\mathcal{P}(y, y)=\mathcal{P}(x, y)$;

(2P) $\mathcal{P}(x, x) \leq \mathcal{P}(x, y)$;

(3P) $\mathcal{P}(x, y)=\mathcal{P}(y, x)$.

Then, the pair $(X, \mathcal{P})$ is said to be a partial symmetric space.

A partial symmetric space $(X, \mathcal{P})$ reduces to a symmetric space if $\mathcal{P}(x, x)=0$, for all $x \in X$. Obviously, every symmetric space is a partial symmetric space, but not conversely.

Example 1. Let $X=\mathbb{R}$ and define a mapping $\mathcal{P}: X \times X \rightarrow \mathbb{R}_{+}$for all $x, y \in X$ and $p, q>1$, as follows:

$$
\mathcal{P}(x, y)=|x-y|^{p}+|x-y|^{q} .
$$

Then, the pair $(X, \mathcal{P})$ is a partial symmetric space.

Example 2. Let $X=\mathbb{R}_{+}$and define a mapping $\mathcal{P}: X \times X \rightarrow \mathbb{R}_{+}$for all $x, y \in X$ and $p, q>1$, as follows:

$$
\mathcal{P}(x, y)=(\max \{x, y\})^{p}+(\max \{x, y\})^{q} .
$$

Then, the pair $(X, \mathcal{P})$ is a partial symmetric space.

Example 3. Let $X=[0, \pi)$ and define a mapping $\mathcal{P}: X \times X \rightarrow \mathbb{R}_{+}$for all $x, y \in X$ and $\alpha>0$, as follows:

$$
\mathcal{P}(x, y)=\sin |x-y|+\alpha .
$$

Then, the pair $(X, \mathcal{P})$ is a partial symmetric space.

Let $(X, \mathcal{P})$ be a partial symmetric space. Then, the $\mathcal{P}$-open ball, with center $x \in X$ and radius $\epsilon>0$, is defined by:

$$
B_{\mathcal{P}}(x, \epsilon)=\{y \in X: \mathcal{P}(x, y)<\mathcal{P}(x, x)+\epsilon\} .
$$

Similarly, the $\mathcal{P}$-closed ball, with center $x \in X$ and radius $\epsilon>0$, is defined by:

$$
B_{\mathcal{P}}[x, \epsilon]=\{y \in X: \mathcal{P}(x, y) \leq \mathcal{P}(x, x)+\epsilon\} .
$$

The family of $\mathcal{P}$-open balls for all $x \in X$ and $\epsilon>0$,

$$
\mathcal{U}_{\mathcal{P}}=\left\{B_{\mathcal{P}}(x, \epsilon): x \in X, \epsilon>0\right\},
$$


forms a basis of some topology $\tau_{\mathcal{P}}$ on $X$.

Lemma 1. Let $\left(X, \tau_{\mathcal{P}}\right)$ be a topological space and $f: X \rightarrow X$. If $f$ is continuous then, for every convergent sequence $x_{n} \rightarrow x$ in $X$, the sequence $f x_{n}$ converges to $f x$. The converse holds if $X$ is metrizable.

In subsequent future discussions, we need some more basic definitions, namely: Convergent sequences, Cauchy sequences, and complete partial symmetric spaces, which are outlined in the following:

Definition 2. A sequence $\left\{x_{n}\right\}$ in $(X, \mathcal{P})$ is said to be $\mathcal{P}$-convergent to $x \in X$, with respect to $\tau_{\mathcal{P}}$, if

$$
\mathcal{P}(x, x)=\lim _{n \rightarrow \infty} \mathcal{P}\left(x_{n}, x\right)
$$

Definition 3. A sequence $\left\{x_{n}\right\}$ in $(X, \mathcal{P})$ is said to be $\mathcal{P}$-Cauchy if and only if $\lim _{n, m \rightarrow \infty} \mathcal{P}\left(x_{n}, x_{m}\right)$ exists and is finite.

Definition 4. A partial symmetric space $(X, \mathcal{P})$ is said to be $\mathcal{P}$-complete if every $\mathcal{P}$-Cauchy sequence $\left\{x_{n}\right\}$ in $X$ is $\mathcal{P}$-convergent, with respect to $\tau_{\mathcal{P}}$ to a point in $x \in X$, such that

$$
\mathcal{P}(x, x)=\lim _{n \rightarrow \infty} \mathcal{P}\left(x_{n}, x\right)=\lim _{n, m \rightarrow \infty} \mathcal{P}\left(x_{n}, x_{m}\right)
$$

Now, we adopt some definitions from symmetric spaces in the setting of partial symmetric spaces:

Definition 5. Let $(X, \mathcal{P})$ be a partial symmetric. Then

(A1) $\lim _{n \rightarrow \infty} \mathcal{P}\left(x_{n}, x\right)=\mathcal{P}(x, x)$ and $\lim _{n \rightarrow \infty} \mathcal{P}\left(x_{n}, y\right)=\mathcal{P}(x, y)$ imply that $x=y$, for a sequence $\left\{x_{n}\right\}, x$, and $y$ in $X$.

(A2) A partial symmetric $\mathcal{P}$ is said to be 1 -continuous if $\lim _{n \rightarrow \infty} \mathcal{P}\left(x_{n}, x\right)=\mathcal{P}(x, x)$ implies that $\lim _{n \rightarrow \infty} \mathcal{P}\left(x_{n}, y\right)=\mathcal{P}(x, y)$, where $\left\{x_{n}\right\}$ is a sequence in $X$ and $x, y \in X$.

(A3) A partial symmetric $\mathcal{P}$ is said to be continuous if $\lim _{n \rightarrow \infty} \mathcal{P}\left(x_{n}, x\right)=\mathcal{P}(x, x)$ and $\lim _{n \rightarrow \infty} \mathcal{P}\left(x_{n}, y\right)=\mathcal{P}(x, y)$ imply that $\lim _{n \rightarrow \infty} \mathcal{P}\left(x_{n}, y_{n}\right)=\mathcal{P}(x, y)$ where $\left\{x_{n}\right\}$ and $\left\{y_{n}\right\}$ are sequences in $X$ and $x, y \in X$.

(A4) $\lim _{n \rightarrow \infty} \mathcal{P}\left(x_{n}, x\right)=\mathcal{P}(x, x)$ and $\lim _{n \rightarrow \infty} \mathcal{P}\left(x_{n}, y_{n}\right)=\mathcal{P}(x, x)$ imply $\lim _{n \rightarrow \infty} \mathcal{P}\left(y_{n}, x\right)=\mathcal{P}(x, x)$, for sequences $\left\{x_{n}\right\},\left\{y_{n}\right\}$, and $x$ in $X$.

(A5) $\lim _{n \rightarrow \infty} \mathcal{P}\left(x_{n}, y_{n}\right)=\mathcal{P}(x, x)$ and $\lim _{n \rightarrow \infty} \mathcal{P}\left(y_{n}, z_{n}\right)=\mathcal{P}(x, x)$ imply $\lim _{n \rightarrow \infty} \mathcal{P}\left(x_{n}, z_{n}\right)=\mathcal{P}(x, x)$, for sequences $\left\{x_{n}\right\},\left\{y_{n}\right\},\left\{z_{n}\right\}$, and $x$ in $X$.

Remark 1. From the Definition 5 , it is observed that $(A 3) \Rightarrow(A 2),(A 4) \Rightarrow(A 1)$, and $(A 2) \Rightarrow(A 1)$ but, in general, the converse implications are not true.

\section{Fixed Point Results}

Let $(X, \mathcal{P})$ be a partial symmetric space and $f: X \rightarrow X$. Then, for every $x \in X$ and for all $i, j \in \mathbb{N}$, we define

$$
\mathfrak{S}(\mathcal{P}, f, x)=\sup \left\{\mathcal{P}\left(f^{i} x, f^{j} x\right): i, j \in \mathbb{N}\right\}
$$

Definition 6. Let $(X, \mathcal{P})$ be a partial symmetric space. A mapping $f: X \rightarrow X$ is said to be a $\kappa$-contraction if

$$
\mathcal{P}(f x, f y) \leq \kappa \mathcal{P}(x, y), \quad \forall x, y \in X
$$

where $\kappa \in(0,1)$. 
Now, we prove an analogue of the Banach contraction principle in the setting of partial symmetric spaces:

Theorem 1. Let $(X, \mathcal{P})$ be a complete partial symmetric space and $f: X \rightarrow X$. Assume that the following conditions are satisfied:

(i) $\quad f$ is a $\kappa$-contraction, for some $\kappa \in[0,1)$;

(ii) there exists $x_{0} \in X$ such that $\mathfrak{S}\left(\mathcal{P}, f, x_{0}\right)<\infty$; and

(iii) either

(a) $f$ is continuous, or

(b) $(X, \mathcal{P})$ satisfies the $(A 1)$ property.

Then, $f$ has a unique fixed point $x \in X$ such that $\mathcal{P}(x, x)=0$.

Proof. Choose $x_{0} \in X$ and construct an iterative sequence $\left\{x_{n}\right\}$ by:

$$
x_{1}=f x_{0}, x_{2}=f^{2} x_{0}, x_{3}=f^{3} x_{0}, \ldots, x_{n}=f^{n} x_{0}, \ldots
$$

Now, from (2) (for all $i, j \in \mathbb{N}$ ), we have

$$
\mathcal{P}\left(f^{n+i} x_{0}, f^{n+j} x_{0}\right) \leq \kappa \mathcal{P}\left(f^{n-1+i} x_{0}, f^{n-1+j} x_{0}\right)
$$

The above inequality holds for all $i, j \in \mathbb{N}$; therefore, by conditions (ii) and (1), we have

$$
\mathfrak{S}\left(\mathcal{P}, f, f^{n} x_{0}\right) \leq \kappa \mathfrak{S}\left(\mathcal{P}, f, f^{n-1} x_{0}\right) .
$$

Repeating this procedure indefinitely, we have (for every $n \in \mathbb{N}$ )

$$
\mathfrak{S}\left(\mathcal{P}, f, f^{n} x_{0}\right) \leq \kappa^{n} \mathfrak{S}\left(\mathcal{P}, f, x_{0}\right)
$$

Let $n, m \in \mathbb{N}$, such that $m=n+p$ for some $p \in \mathbb{N}$. Using (3), we have

$$
\mathcal{P}\left(f^{n} x_{0}, f^{n+p} x_{0}\right) \leq \mathfrak{S}\left(\mathcal{P}, f, f^{n} x_{0}\right) \leq \kappa^{n} \mathfrak{S}\left(\mathcal{P}, f, x_{0}\right) .
$$

As $\mathfrak{S}\left(\mathcal{P}, f, x_{0}\right)<\infty$ and $\kappa \in(0,1)$, we have

$$
\lim _{n, m \rightarrow \infty} \mathcal{P}\left(x_{n}, x_{m}\right)=0,
$$

so that $\left\{x_{n}\right\}$ is a $\mathcal{P}$-Cauchy sequence in $X$. In light of the $\mathcal{P}$-completeness of $X$, there exists $x \in X$ such that $\left\{x_{n}\right\} \mathcal{P}$-converges to $x$. Now, we show that $x \in X$ is a fixed point of $f$.

Assume that $f$ is continuous. Then,

$$
x=\lim _{n \rightarrow \infty} x_{n+1}=f\left(\lim _{n \rightarrow \infty} x_{n}\right)=f x .
$$

Alternately, assume that $(X, \mathcal{P})$ satisfies the $(A 1)$ property. Now, we have

$$
\mathcal{P}\left(f x_{n}, f x\right) \leq \mathcal{P}\left(x_{n}, x\right),
$$

which, on taking $n \rightarrow \infty$, implies that $\lim _{n \rightarrow \infty} \mathcal{P}\left(x_{n+1}, f x\right)=0$. Thus, from the $(A 1)$ property, $f x=x$. Therefore, $x$ is a fixed point of $f$. To prove the uniqueness of the fixed point, let on contrary that there exist $x, y \in X$ such that $f x=x$ and $f y=y$. Then, by the definition of $\kappa$-contraction, we have

$$
\mathcal{P}(x, y)=\mathcal{P}(f x, f y) \leq \kappa \mathcal{P}(x, y),
$$


a contradiction. Hence, $x=y$; that is, $x$ is a unique fixed point of $f$. Finally, we show that $\mathcal{P}(x, x)=0$. Since, $f$ is $k$-contraction mapping, we have

$$
\mathcal{P}(x, x)=\mathcal{P}(f x, f x) \leq \kappa \mathcal{P}(x, x) .
$$

This implies that $\mathcal{P}(x, x)<0$, implying thereby that $\mathcal{P}(x, x)=0$. This completes the proof.

Now, we recall the definition of the Kannan-Ćirić contraction condition [14]:

Definition 7. Let $(X, \mathcal{P})$ be a partial symmetric space. A mapping $f: X \rightarrow X$ is said to be a Kannan-Ćirić type $\kappa$-contraction if, for all $x, y \in X$,

$$
\mathcal{P}(f x, f y) \leq \kappa \max \{\mathcal{P}(x, f x), \mathcal{P}(y, f y)\}
$$

where $\kappa \in[0,1)$.

Next, we prove a fixed point result via Kannan-Ćirić type $\kappa$-contractions in the setting of partial symmetric spaces:

Theorem 2. Let $(X, \mathcal{P})$ be a complete partial symmetric space and $f: X \rightarrow X$. Assume that the following conditions are satisfied:

(i) $f$ is a Kannan-Ćirić type $\kappa$-contraction mapping,

(ii) $f$ is continuous.

Then, $f$ has a unique fixed point $x \in X$ such that $\mathcal{P}(x, x)=0$.

Proof. Take $x_{0} \in X$, and construct an iterative sequence $\left\{x_{n}\right\}$ by:

$$
x_{1}=f x_{0}, x_{2}=f^{2} x_{0}, x_{3}=f^{3} x_{0}, \ldots, x_{n}=f^{n} x_{0}, \ldots
$$

Now, we assert that $\lim _{n \rightarrow \infty} \mathcal{P}\left(x_{n}, x_{n+1}\right)=0$. On setting $x=x_{n}$ and $y=x_{n+1}$ in (4), we get

$$
\begin{aligned}
\mathcal{P}\left(x_{n}, x_{n+1}\right) & =\mathcal{P}\left(f x_{n-1}, f x_{n}\right) \\
& \leq \kappa \max \left\{\mathcal{P}\left(x_{n-1}, f x_{n-1}\right), \mathcal{P}\left(x_{n}, f x_{n}\right)\right\} \\
& \leq \kappa \max \left\{\mathcal{P}\left(x_{n-1}, x_{n}\right), \mathcal{P}\left(x_{n}, x_{n+1}\right)\right\}
\end{aligned}
$$

Assume that $\max \left\{\mathcal{P}\left(x_{n-1}, x_{n}\right), \mathcal{P}\left(x_{n}, x_{n+1}\right)\right\}=\mathcal{P}\left(x_{n}, x_{n+1}\right)$, then from (5), we have

$$
\mathcal{P}\left(x_{n}, x_{n+1}\right) \leq \kappa \mathcal{P}\left(x_{n}, x_{n+1}\right),
$$

a contradiction (since $\kappa \in(0,1))$. Thus, $\max \left\{\mathcal{P}\left(x_{n-1}, x_{n}\right), \mathcal{P}\left(x_{n}, x_{n+1}\right)\right\}=\mathcal{P}\left(x_{n-1}, x_{n}\right)$. Therefore, (5) gives rise

$$
\mathcal{P}\left(x_{n}, x_{n+1}\right)=\kappa \mathcal{P}\left(x_{n-1}, x_{n}\right) \text { for all } n \in \mathbb{N} .
$$

Thus, inductively, we have

$$
\mathcal{P}\left(x_{n}, x_{n+1}\right)=\kappa^{n} \mathcal{P}\left(x_{0}, x_{1}\right) \text { for all } n \in \mathbb{N} .
$$

On taking the limit as $n \rightarrow \infty$, we get

$$
\lim _{n \rightarrow \infty} \mathcal{P}\left(x_{n}, x_{n+1}\right)=0 .
$$


Now, we assert that $\left\{x_{n}\right\}$ is a $\mathcal{P}$-Cauchy sequence. From (4), we have, for $n, m \in \mathbb{N}$,

$$
\begin{aligned}
\mathcal{P}\left(x_{n}, x_{m}\right) & =\mathcal{P}\left(f x_{n-1}, f x_{m-1}\right) \\
& \leq \kappa \max \left\{\mathcal{P}\left(x_{n-1}, f x_{n-1}\right), \mathcal{P}\left(x_{m-1}, f x_{m-1}\right)\right\} \\
& \leq \kappa \max \left\{\mathcal{P}\left(x_{n-1}, x_{n}\right), \mathcal{P}\left(x_{m-1}, x_{m}\right)\right\} .
\end{aligned}
$$

By taking the limit as $n, m \rightarrow \infty$ and using (6), we have

$$
\lim _{n, m \rightarrow \infty} \mathcal{P}\left(x_{n}, x_{m}\right)=0
$$

Hence, $\left\{x_{n}\right\}$ is a $\mathcal{P}$-Cauchy sequence. Since $(X, \mathcal{P})$ is $\mathcal{P}$-complete, there exists $x \in X$ such that $\lim _{n \rightarrow \infty} \mathcal{P}\left(x_{n}, x\right)=0$. Now, we show that $x \in X$ is a fixed point of $f$. By the continuity of $f$, we have

$$
x=\lim _{n \rightarrow \infty} x_{n+1}=f\left(\lim _{n \rightarrow \infty} x_{n}\right)=f x .
$$

Therefore, $x$ is a fixed point of $f$. For the uniqueness part, let on contrary that there exist $x, y \in X$ such that $f x=x$ and $f y=y$. Then, from (4), we have

$$
\begin{aligned}
\mathcal{P}(x, y) & =\mathcal{P}(f x, f y) \\
& \leq \kappa \max \{\mathcal{P}(x, f x), \mathcal{P}(y, f y)\} \\
& \leq \kappa \max \{\mathcal{P}(x, x), \mathcal{P}(y, y)\} .
\end{aligned}
$$

So, either $\mathcal{P}(x, y) \leq \kappa \mathcal{P}(x, x)$ or $\mathcal{P}(x, y) \leq \kappa \mathcal{P}(y, y)$, which is a contradiction. Therefore, $x$ is a unique fixed point of $f$. Finally, we show that $\mathcal{P}(x, x)=0$. From (4), we have

$$
\begin{aligned}
\mathcal{P}(x, x) & =\kappa \mathcal{P}(f x, f x) \\
& \leq \kappa \max \{\mathcal{P}(x, f x), \mathcal{P}(x, f x)\}, \\
& \leq \kappa \max \{\mathcal{P}(x, x), \mathcal{P}(x, x)\}, \\
& \leq \kappa \mathcal{P}(x, x),
\end{aligned}
$$

this implies that $\mathcal{P}(x, x)<0$, implying thereby that $\mathcal{P}(x, x)=0$. This completes the proof.

Now, we present some fixed point results for Ćirić quasi contractions in the setting of partial symmetric spaces. We start with the following definition.

Definition 8. Let $(X, \mathcal{P})$ be a partial symmetric space and $f: X \rightarrow X$. Then $f$ is said to be $\kappa$-weak contraction if, for all $x, y \in X$, and $\kappa \in(0,1)$

$$
\mathcal{P}(f x, f y) \leq \kappa \max \{\mathcal{P}(x, y), \mathcal{P}(x, f x), \mathcal{P}(y, f y), \mathcal{P}(x, f y), \mathcal{P}(y, f x)\}
$$

Proposition 1. Let $f$ be a $\kappa$-weak contraction for any $\kappa \in(0,1)$. If $x$ is a fixed point of $f$, then $\mathcal{P}(x, x)=0$.

Proof. Suppose $x \in X$ is a fixed point of $f$. Since $f$ is a $\kappa$-weak contraction, we have that

$$
\begin{aligned}
\mathcal{P}(x, x) & =\mathcal{P}(f x, f x) \\
\leq & \kappa \max \{\mathcal{P}(x, x), \mathcal{P}(x, f x), \mathcal{P}(x, f x), \mathcal{P}(x, f x), \mathcal{P}(x, f x)\} \\
& =\kappa \max \{\mathcal{P}(x, x), \mathcal{P}(x, x), \mathcal{P}(x, x), \mathcal{P}(x, x), \mathcal{P}(x, x)\} \\
& =\kappa \mathcal{P}(x, x),
\end{aligned}
$$

this implies that $\mathcal{P}(x, x)<0$, implying thereby $\mathcal{P}(x, x)=0$. 
Theorem 3. Let $(X, \mathcal{P})$ be a $\mathcal{P}$-complete partial symmetric space and $f: X \rightarrow X$. Suppose that the following conditions hold:

(i) $f$ is a $\kappa$-weak contraction for some $\kappa \in[0,1)$;

(ii) there exists $x_{0} \in X$ such that $\mathfrak{S}(\mathcal{P}, f, x)<\infty$; and

(iii) $f$ is continuous.

Then, $f$ has a unique fixed point.

Proof. Assume $x_{0} \in X$, and construct an iterative sequence $\left\{x_{n}\right\}$ by:

$$
x_{1}=f x_{0}, x_{2}=f^{2} x_{0}, x_{3}=f^{3} x_{0}, \ldots, x_{n}=f^{n} x_{0}, \ldots
$$

Let $n$ be an arbitrary positive integer. Since $f$ is a $\kappa$-weak contraction, for all $i, j \in \mathbb{N}$, we have

$$
\begin{aligned}
\mathcal{P}\left(f^{n+i} x_{0}, f^{n+j} x_{0}\right) \leq & \kappa \max \left\{\mathcal{P}\left(f^{n-1+i} x_{0}, f^{n-1+j} x_{0}\right), \mathcal{P}\left(f^{n-1+i} x_{0}, f^{n+i} x_{0}\right),\right. \\
& \left.\mathcal{P}\left(f^{n-1+j} x_{0}, f^{n+j} x_{0}\right), \mathcal{P}\left(f^{n-1+i} x_{0}, f^{n+j} x_{0}\right), \mathcal{P}\left(f^{n-1+j} x_{0}, f^{n+i} x_{0}\right)\right\} .
\end{aligned}
$$

Since the above inequality is true for all $i, j \in \mathbb{N}$, therefore by conditions (ii) and (1), we have

$$
\mathfrak{S}\left(\mathcal{P}, f, f^{n} x_{0}\right) \leq \kappa \mathfrak{S}\left(\mathcal{P}, f, f^{n-1} x_{0}\right) .
$$

Continuing this process indefinitely, we have, for all $n \geq 1$,

$$
\mathfrak{S}\left(\mathcal{P}, f, f^{n} x_{0}\right) \leq \kappa^{n} \mathfrak{S}\left(\mathcal{P}, f, x_{0}\right)
$$

Now, for each $n, m \in \mathbb{N}$, such that $m=n+p$ for some $p \in \mathbb{N}$, we have, due to (9), that

$$
\mathcal{P}\left(f^{n} x_{0}, f^{n+p} x_{0}\right) \leq \mathfrak{S}\left(\mathcal{P}, f, f^{n} x_{0}\right) \leq \kappa^{n} \mathfrak{S}\left(\mathcal{P}, f, x_{0}\right) .
$$

Since $\mathfrak{S}\left(\mathcal{P}, f, x_{0}\right)<\infty$ and $\kappa \in(0,1)$, we have

$$
\lim _{n, m \rightarrow \infty} \mathcal{P}\left(x_{n}, x_{m}\right)=0,
$$

so $\left\{x_{n}\right\}$ is a $\mathcal{P}$-Cauchy sequence in $X$. In view of the $\mathcal{P}$-completeness of $X$, there exists $x \in X$ such that $\left\{x_{n}\right\} \mathcal{P}$-converges to $x$. Now, we show that $x$ is a fixed point of $f$. By the continuity of $f$, we have

$$
x=\lim _{n \rightarrow \infty} x_{n+1}=f\left(\lim _{n \rightarrow \infty} x_{n}\right)=f x .
$$

Therefore, $x$ is a fixed point of $f$. For the uniqueness part, let on contrary that there exist $x, y \in X$ such that $f x=x$ and $f y=y$. Thus, by using the condition (8), we have

$$
\begin{aligned}
\mathcal{P}(x, y) & =\mathcal{P}(f x, f y) \\
& \leq \kappa \max \{\mathcal{P}(x, y), \mathcal{P}(x, f x), \mathcal{P}(y, f y), \mathcal{P}(x, f y), \mathcal{P}(y, f x)\} \\
& =\kappa \max \{\mathcal{P}(x, y), \mathcal{P}(x, x), \mathcal{P}(y, y), \mathcal{P}(x, y), \mathcal{P}(y, x)\}
\end{aligned}
$$

By using the property $(2 \mathcal{P})$, we have

$$
\mathcal{P}(x, y) \leq \kappa \mathcal{P}(x, y)<\mathcal{P}(x, y),
$$

a contradiction, and so $\mathcal{P}(x, y)=0$; which implies that $x=y$. Thus, $f$ has a unique fixed point. This completes the proof.

Now, we furnish the following example, which illustrates Theorem 3. 
Example 4. Consider $X=[0,1]$ and a partial symmetric $\mathcal{P}: X \times X \rightarrow \mathbb{R}_{+}$defined by $\mathcal{P}(x, y)=\max \{x, y\}$, for all $x, y \in X$. Define a self-mapping $f$ on $X$ by

$$
f x=\frac{2 x^{2}}{5}, \text { for all } x \in X .
$$

Observe that

$$
\begin{aligned}
\mathcal{P}(f x, f y) & =\max \{f x, f y\} \\
& =\max \left\{\frac{2 x^{2}}{5}, \frac{2 y^{2}}{5}\right\} \\
& \leq \frac{2}{5} \max \{x, y\}=\frac{2}{5} \mathcal{P}(x, y) \\
& \leq \frac{2}{5} \max \{\mathcal{P}(x, y), \mathcal{P}(x, f x), \mathcal{P}(y, f y), \mathcal{P}(x, f y), \mathcal{P}(y, f x)\},
\end{aligned}
$$

for all $x, y \in X$. Observe that $f$ is continuous and condition (ii) holds. Thus, all the conditions of Theorem 3 are satisfied and so $f$ has a unique fixed point (i.e., $x=0$ ).

Notice that this example can not be covered by metrical fixed point theorems.

Corollary 1. The conclusions of Theorem 3 remain true, if the contractive condition (8) is replaced by any one of the following:

(i) $\mathcal{P}(f x, f y) \leq \frac{\kappa}{2}[\mathcal{P}(x, f y)+\mathcal{P}(y, f x)]$;

(ii) $\mathcal{P}(f x, f y) \leq \kappa \max \{\mathcal{P}(x, y), \mathcal{P}(x, f x), \mathcal{P}(y, f y)\}$;

(iii) $\mathcal{P}(f x, f y) \leq \kappa \max \{\mathcal{P}(x, y), \mathcal{P}(x, f y), \mathcal{P}(y, f x)\}$;

(iv) $\mathcal{P}(f x, f y) \leq \kappa \max \left\{\mathcal{P}(x, y), \frac{\mathcal{P}(x, f x)+\mathcal{P}(y, f y)}{2}, \frac{\mathcal{P}(x, f y)+\mathcal{P}(y, f x)}{2}\right\}$;

(v) $\mathcal{P}(f x, f y) \leq \kappa \max \left\{\mathcal{P}(x, y), \frac{\mathcal{P}(x, f x)+\mathcal{P}(y, f y)}{2}, \mathcal{P}(x, f y), \mathcal{P}(y, f x)\right\}$; or

(vi) $\mathcal{P}(f x, f y) \leq \kappa \max \left\{\mathcal{P}(x, y), \mathcal{P}(x, f x), \mathcal{P}(y, f y), \frac{\mathcal{P}(x, f y)+\mathcal{P}(y, f x)}{2}\right\}$.

\section{Application}

In this section, we endeavor to apply Theorem 1 to prove the existence and uniqueness of a solution of the following integral equation of Fredholm type:

$$
x(t)=\int_{a}^{b} G(t, s, x(s)) d s+h(t) \text { for all } t, s \in[a, b],
$$

where $G, h \in C([a, b], \mathbb{R})$ (say, $X=C([a, b], \mathbb{R})$. Define a partial symmetric space $\mathcal{P}$ on $X$ :

$$
\mathcal{P}(x, y)=\sup _{t \in[a, b]}|x(t)-y(t)|^{p}+\sup _{t \in[a, b]}|x(t)-y(t)|^{q}, \text { for all } x, y \in X, \text { and } p, q>1 .
$$

Then, $(X, \mathcal{P})$ is a complete partial symmetric space.

Now we are equipped to state and prove our result, as follows:

Theorem 4. Assume that, for all $x, y \in C([a, b], \mathbb{R})$,

$$
|G(t, s, x(s))-G(t, s, y(s))| \leq \frac{1}{2(b-a)}|x(s)-y(s)|,
$$


for all $t, s \in[a, b]$. Then, Equation (11) has a unique solution.

Proof. Define $f: X \rightarrow X$ by

$$
f x(t)=\int_{a}^{b} G(t, s, x(s)) d s+h(t) \text { for all } t, s \in[a, b] .
$$

It is clear that $x$ is a fixed point of the operator $f$ if and only if it is a solution of Equation (11). Now, for all $x, y \in X$, we have

$$
\begin{aligned}
|f x(t)-f y(t)|^{p}+|f x(t)-f y(t)|^{q} \leq & \left(\int_{a}^{b}|G(t, s, x(s))-G(t, s, y(s))| d s\right)^{p} \\
& +\left(\int_{a}^{b}|G(t, s, x(s))-G(t, s, y(s))| d s\right)^{q} \\
\leq & \left(\int_{a}^{b} \frac{1}{2(b-a)}|x(s)-y(s)| d s\right)^{p} \\
& +\left(\int_{a}^{b} \frac{1}{2(b-a)}|x(s)-y(s)| d s\right)^{q} \\
\leq & \frac{1}{2^{p}(b-a)^{p}} \sup _{t \in[a, b]}|x(t)-y(t)|^{p}\left(\int_{a}^{b} d s\right)^{p} \\
& +\frac{1}{2^{q}(b-a)^{q}} \sup _{t \in[a, b]}|x(t)-y(t)|^{q}\left(\int_{a}^{b} d s\right)^{q} \\
\leq & \lambda\left(\sup _{t \in[a, b]}|x(t)-y(t)|^{p}+\sup _{t \in[a, b]}|x(t)-y(t)|^{q}\right) .
\end{aligned}
$$

Thus, condition (12) is satisfied, with $\lambda=\max \left\{\frac{1}{2^{p}}, \frac{1}{2^{q}}\right\} \in[0,1)$. Hence, the operator $f$ has a unique fixed point; that is, the Fredholm integral Equation (11) has a unique solution.

\section{Results Involving Set-Valued Map}

In this section, first we extend the idea of Hausdorff distance to partial symmetric spaces. Let $(X, \mathcal{P})$ be a partial symmetric space and $\mathcal{C B}^{\mathcal{P}}(X)$ be the family of all nonempty, $\tau_{\mathcal{P}}$-closed, and bounded subsets of $(X, \mathcal{P})$. Observe that $A$ will be bounded if there exist $x_{0} \in X$ and $M \geq 0$ such that, for all $a \in A, \mathcal{P}\left(x_{0}, a\right) \leq \mathcal{P}(a, a)+M$.

Moreover, for $A, B \in \mathcal{C B}^{\mathcal{P}}(X)$ and $x \in X$, we define:

$$
\begin{gathered}
\operatorname{dist}_{\mathcal{P}}(x, A)=\inf \{\mathcal{P}(x, a): a \in A\} ; \\
\delta_{\mathcal{P}}(A, B)=\sup \left\{\operatorname{dist}_{\mathcal{P}}(a, B): a \in A\right\} ; \text { and } \\
\delta_{\mathcal{P}}(B, A)=\sup \left\{\operatorname{dist}_{\mathcal{P}}(b, A): b \in B\right\} .
\end{gathered}
$$

Remark 2. Let $(X, \mathcal{P})$ be a partial symmetric space and $A$ a non-empty subset of $X$, then

$$
a \in \bar{A} \text { if and only if } \operatorname{dist}_{\mathcal{P}}(a, A)=\mathcal{P}(a, a)
$$

where $\bar{A}$ denotes the closure of $A$, with respect to the partial symmetric $\mathcal{P}$. Also, $A$ is $\mathcal{P}$-closed in $(X, \mathcal{P})$ if and only if $A=\bar{A}$.

Proposition 2. Let $(X, \mathcal{P})$ be a partial symmetric space. For $A, B, C \in \mathcal{C B}^{\mathcal{P}} X$, we have the following:

(i) $\delta_{\mathcal{P}}(A, A)=\sup \{\mathcal{P}(a, a): a \in A\}$;

(ii) $\delta_{\mathcal{P}}(A, A) \leq \delta_{\mathcal{P}}(A, B)$;

(iii) $B \subset C \Rightarrow \delta_{\mathcal{P}}(A, C) \leq \delta_{\mathcal{P}}(A, B)$; 
(iv) $\delta_{\mathcal{P}}(A, B)=0 \Rightarrow A \subseteq B$; and

(v) $\delta_{\mathcal{P}}(A \cup B, C)=\max \left\{\delta_{\mathcal{P}}(A, C), \delta_{\mathcal{P}}(B, C)\right\}$.

Proof. (i) Suppose $A \in \mathcal{C B}^{\mathcal{P}}(X)$. Since $a \in \bar{A}$ if and only if $\mathcal{P}(a, A)=\mathcal{P}(a, a)$,

$$
\delta_{\mathcal{P}}(A, A)=\sup \left\{\operatorname{dist}_{\mathcal{P}}(a, A): a \in A\right\}=\sup \{\mathcal{P}(a, a): a \in A\} .
$$

(ii) Suppose $a \in A$. By definition of the partial symmetric space, we know that $\mathcal{P}(a, a) \leq \mathcal{P}(a, b)$, which implies that

$$
\mathcal{P}(a, a) \leq \operatorname{dist}_{\mathcal{P}}(a, B) \leq \delta_{\mathcal{P}}(A, B) .
$$

Hence, condition $(i)$ gives rise to

$$
\delta_{\mathcal{P}}(A, A) \leq \delta_{\mathcal{P}}(A, B)
$$

(iii) Suppose $A, B, C \in \mathcal{C B}^{\mathcal{P}}(X)$, such that $B \subseteq C$. Then,

$$
\operatorname{dist}_{\mathcal{P}}(x, B) \leq \operatorname{dist}_{\mathcal{P}}(x, C) \text { for all } a \in X .
$$

Thus,

$$
B \subset C \Rightarrow \delta_{\mathcal{P}}(A, C) \leq \delta_{\mathcal{P}}(A, B) .
$$

(iv) Suppose $A, B \in \mathcal{C B}^{\mathcal{P}}(X)$, such that $\delta_{\mathcal{P}}(A, B)=0$. Then,

$$
\sup \left\{\operatorname{dist}_{\mathcal{P}}(a, A): a \in A\right\}=0 \Rightarrow \operatorname{dist}_{\mathcal{P}}(a, B)=0 \text { for all } a \in A .
$$

In view of the above conditions $(i)$ and (ii), we have

$$
\mathcal{P}(a, a) \leq \delta_{\mathcal{P}}(A, B)=0 \Rightarrow \mathcal{P}(a, a)=0, \text { for all } a \in A .
$$

Therefore, $\operatorname{dist}_{\mathcal{P}}(a, B)=\mathcal{P}(a, a)$ for all $a \in A$ implies that ' $a$ ' is in the closure of $B$ for all $a \in A$. Since $B$ is $\mathcal{P}$-closed, we have $A \subseteq B$.

(v) Suppose $A, B, C \in \mathcal{C B}^{\mathcal{P}}(X)$. Then,

$$
\begin{aligned}
\delta_{\mathcal{P}}(A \cup B, C) & =\sup \left\{\operatorname{dist}_{\mathcal{P}}(x, C): x \in A \cup B\right\} \\
& =\max \left\{\sup \left\{\operatorname{dist}_{\mathcal{P}}(x, C): x \in A\right\}, \sup \left\{\operatorname{dist}_{\mathcal{P}}(x, C): x \in B\right\}\right\} \\
& =\max \left\{\delta_{\mathcal{P}}(A, C), \delta_{\mathcal{P}}(B, C)\right\} .
\end{aligned}
$$

Next, let $(X, \mathcal{P})$ be a partial symmetric space. Define

$$
H_{\mathcal{P}}(A, B)=\max \left\{\delta_{\mathcal{P}}(A, B), \delta_{\mathcal{P}}(B, A)\right\} .
$$

Proposition 3. Let $(X, \mathcal{P})$ be a partial symmetric space. For $A, B, C, D \in \mathcal{C B}^{\mathcal{P}}(X)$, we have the following:

(1H) $\quad H_{\mathcal{P}}(A, A) \leq H_{\mathcal{P}}(A, B)$;

(2H) $\quad H_{\mathcal{P}}(A, B)=H_{\mathcal{P}}(B, A)$; and

(3H) $\quad H_{\mathcal{P}}(A \cup B, C \cup D)=\max \left\{H_{\mathcal{P}}(A, C), H_{\mathcal{P}}(B, D)\right\}$.

Proof. $(1 H)$ By condition (ii) of Proposition 2, we have

$$
H_{\mathcal{P}}(A, A)=\delta_{\mathcal{P}}(A, A) \leq \delta_{\mathcal{P}}(A, B) \leq H_{\mathcal{P}}(A, B) .
$$


(2H) By the definition of $H_{\mathcal{P}}$, we have

$$
H_{\mathcal{P}}(A, B)=\max \left\{\delta_{\mathcal{P}}(A, B), \delta_{\mathcal{P}}(B, A)\right\}=\max \left\{\delta_{\mathcal{P}}(B, A), \delta_{\mathcal{P}}(A, B)\right\}=H_{\mathcal{P}}(B, A) .
$$

$(3 H)$ By condition $(v)$ of Proposition 2, we have

$$
\begin{aligned}
\delta_{\mathcal{P}}(A \cup B, C \cup D) & =\max \left\{\delta_{\mathcal{P}}(A, C \cup D), \delta_{\mathcal{P}}(B, C \cup D)\right\} \\
& \leq \max \left\{\delta_{\mathcal{P}}(A, C), \delta_{\mathcal{P}}(B, D)\right\} \\
& \leq \max \left\{H_{\mathcal{P}}(A, C), H_{\mathcal{P}}(B, D)\right\} .
\end{aligned}
$$

Similarly, we obtain

$$
\delta_{\mathcal{P}}(C \cup D, A \cup B) \leq \max \left\{H_{\mathcal{P}}(A, C), H_{\mathcal{P}}(B, D)\right\} .
$$

Hence, by the definition of $H_{\mathcal{P}}$, we have, for all $A, B, C, D \in \mathcal{C B}^{\mathcal{P}}(X)$, that

$$
H_{\mathcal{P}}(A \cup B, C \cup D)=\max \left\{H_{\mathcal{P}}(A, C), H_{\mathcal{P}}(B, D)\right\} .
$$

Proposition 4. Let $(X, \mathcal{P})$ be a partial symmetric space. For $A, B \in \mathcal{C B}^{\mathcal{P}}(X)$, we have

$$
H_{\mathcal{P}}(A, B)=0 \Rightarrow A=B .
$$

Proof. Let $H_{\mathcal{P}}(A, B)=0$. Then, by the definition of $H_{\mathcal{P}}$, we have

$$
\delta_{\mathcal{P}}(A, B)=\delta_{\mathcal{P}}(B, A)=0 .
$$

Thus, by condition (iii) of Proposition 2, we get $A \subseteq B$ and $B \subseteq A$, which implies $A=B$.

Now, we prove the following lemma which is needed in the sequel:

Lemma 2. Let $(X, \mathcal{P})$ be partial symmetric space and $A, B \in \mathcal{C B}^{\mathcal{P}}(X)$. Then, for any $h>1$ and $a \in A$, there exists $b \in B$ such that

$$
\mathcal{P}(a, b) \leq h H_{\mathcal{P}}(A, B)
$$

Proof. First, we consider $A=B$. From (i) of Proposition 2,

$$
H_{\mathcal{P}}(A, B)=H_{\mathcal{P}}(A, A)=\delta_{\mathcal{P}}(A, A)=\sup _{a \in A} \mathcal{P}(a, a) .
$$

Observe that, for any $a \in A$ and any $h>1$, we have

$$
\mathcal{P}(a, a) \leq \sup _{a \in A} \mathcal{P}(a, a)=H_{\mathcal{P}}(A, B) \leq h H_{\mathcal{P}}(A, B) .
$$

Consequently, $b=a$ satisfies the inequality (13). Now, let $A \neq B$. Then, there exists $a \in A$ such that

$$
\mathcal{P}(a, b)>h H_{\mathcal{P}}(A, B) \text { for all } b \in B \text {. }
$$

Then,

$$
\inf \{\mathcal{P}(a, b): b \in B\} \geq h H_{\mathcal{P}}(A, B)
$$

so that

$$
\operatorname{dist}_{\mathcal{P}}(a, B) \geq h H_{\mathcal{P}}(A, B) .
$$


Hence,

$$
H_{\mathcal{P}}(A, B) \geq \delta_{\mathcal{P}}(A, B)=\sup _{a \in A} \operatorname{dist}_{\mathcal{P}}(a, B) \geq \operatorname{dist}_{\mathcal{P}}(a, B) \geq h H_{\mathcal{P}}(A, B),
$$

a contradiction, since $h>1$.

Recall that, if $f: X \rightarrow \mathcal{C B}^{\mathcal{P}}(X)$ is a mapping, then an element $x \in X$ is said to be a fixed point of $f$ if $x \in f x$.

Now, we state and prove our main result in this section:

Theorem 5. Let $(X, \mathcal{P})$ be a complete partial symmetric space and $f: X \rightarrow \mathcal{C B}^{\mathcal{P}}(X)$. Assume that the following conditions are satisfied:

(i) there exists $\kappa \in[0,1)$ such that

$$
H_{\mathcal{P}}(f x, f y) \leq \kappa \mathcal{P}(x, y) \text { for all } x, y \in X
$$

(ii) there exists $x_{0} \in X$ such that $\mathfrak{S}\left(\mathcal{P}, f, x_{0}\right)<\infty$; and

(iii) $f$ is continuous.

Then, $f$ has a unique fixed point $x \in X$, such that $\mathcal{P}(x, x)=0$.

Proof. Suppose $x_{0} \in X$ and $x_{1} \in f x_{0}$. From Lemma 2 with $h=\frac{1}{\sqrt{\kappa}}$, there exists $x_{2} \in f x_{1}$ such that $\mathcal{P}\left(x_{1}, x_{2}\right) \leq \frac{1}{\sqrt{\kappa}} H_{\mathcal{P}}\left(f x_{0}, f x_{1}\right)$. Since $H_{\mathcal{P}}\left(f x_{0}, f x_{1}\right) \leq \kappa \mathcal{P}\left(x_{0}, x_{1}\right)$, then $\mathcal{P}\left(x_{1}, x_{2}\right) \leq \sqrt{\kappa} \mathcal{P}\left(x_{0}, x_{1}\right)$. Similarly, for $x_{2} \in f x_{1}$ there exists $x_{3} \in f x_{2}$ such that

$$
\mathcal{P}\left(x_{2}, x_{3}\right) \leq \frac{1}{\sqrt{\kappa}} H_{\mathcal{P}}\left(f x_{1}, f x_{2}\right) \leq \sqrt{\kappa} \mathcal{P}\left(x_{1}, x_{2}\right)
$$

Inductively, we obtain a sequence $\left\{x_{n}\right\}$ in $X$, such that

$$
x_{n+1} \in f x_{n} \text { and } \mathcal{P}\left(x_{n+1}, x_{n}\right) \leq \sqrt{\kappa} \mathcal{P}\left(x_{n}, x_{n-1}\right) \text {, for all } n \in \mathbb{N} \text {. }
$$

By condition $(i)$, for all $i, j \in \mathbb{N}$ we have

$$
\mathcal{P}\left(f^{n+i} x_{0}, f^{n+j} x_{0}\right) \leq \sqrt{\kappa} \mathcal{P}\left(f^{n-1+i} x_{0}, f^{n-1+j} x_{0}\right) .
$$

Therefore, by condition (ii) and (1), we have

$$
\mathfrak{S}\left(\mathcal{P}, f, f^{n} x_{0}\right) \leq \sqrt{\kappa} \mathfrak{S}\left(\mathcal{P}, f, f^{n-1} x_{0}\right) .
$$

Continuing this process, we have, for every $n \in \mathbb{N}$,

$$
\mathfrak{S}\left(\mathcal{P}, f, f^{n} x_{0}\right) \leq(\sqrt{\kappa})^{n} \mathfrak{S}\left(\mathcal{P}, f, x_{0}\right) .
$$

By using (3), we have, for $n, m, p \in \mathbb{N}$ such that $m=n+p$,

$$
\mathcal{P}\left(f^{n} x_{0}, f^{n+p} x_{0}\right) \leq \mathfrak{S}\left(\mathcal{P}, f, f^{n} x_{0}\right) \leq(\sqrt{\kappa})^{n} \mathfrak{S}\left(\mathcal{P}, f, x_{0}\right) .
$$

Since $\mathfrak{S}\left(\mathcal{P}, f, x_{0}\right)<\infty$ and $\kappa \in(0,1)$, then

$$
\lim _{n, m \rightarrow \infty} \mathcal{P}\left(x_{n}, x_{m}\right)=0,
$$


so that $\left\{x_{n}\right\}$ is a $\mathcal{P}$-Cauchy sequence in $X$. In view of the $\mathcal{P}$-completeness of $X$, there exists $x \in X$ such that $\left\{x_{n}\right\} \mathcal{P}$-converges to $x$. Therefore,

$$
\mathcal{P}(x, x)=\lim _{n \rightarrow \infty} \mathcal{P}\left(x_{n}, x\right)=\lim _{n, m \rightarrow \infty} \mathcal{P}\left(x_{n}, x_{m}\right)=0 .
$$

As $H_{\mathcal{P}}\left(f x_{n}, f x\right) \leq \kappa \mathcal{P}\left(x_{n}, x\right)$, implies that

$$
\lim _{n \rightarrow \infty} H_{\mathcal{P}}\left(f x_{n}, f x\right)=0 .
$$

Hence, $x_{n+1} \in f x_{n}$. Therefore,

$$
\operatorname{dist}_{\mathcal{P}}\left(x_{n+1}, f x\right) \leq \delta_{\mathcal{P}}\left(f x_{n}, f x\right) \leq H_{\mathcal{P}}\left(f x_{n}, f x\right) .
$$

Hence,

$$
\lim _{n \rightarrow \infty} \operatorname{dist}_{\mathcal{P}}\left(x_{n+1}, f x\right)=0 .
$$

By the continuity of $f$, we obtain

$$
\operatorname{dist}_{\mathcal{P}}(x, f x)=\lim _{n \rightarrow \infty} \operatorname{dist}_{\mathcal{P}}\left(x_{n+1}, f x\right)=0 .
$$

Thus, we have $\mathcal{P}(x, x)=\operatorname{dist}_{\mathcal{P}}(x, f x)=0$. As $f x$ is $\mathcal{P}$-closed, then we have $x \in f x$. Hence, $x$ is a fixed point of $f$ in $X$. This completes the proof.

Next, we adopt the following example to demonstrate Theorem 5.

Example 5. Consider $X=\{0,1,2\}$ equipped with the partial symmetric $\mathcal{P}: X \times X \rightarrow \mathbb{R}_{+}$defined by

$$
\mathcal{P}(x, y)=\frac{1}{2}|x-y|^{2}+\frac{1}{4}(\max \{x, y\})^{2}, \text { for all } x, y \in X .
$$

Then $(X, \mathcal{P})$ is a $\mathcal{P}$-complete symmetric space. Note that $\{0\}$ and $\{0,1\}$ are bounded sets in $(X, \mathcal{P})$. In fact, if $x \in\{0,1,2\}$ then

$$
\begin{aligned}
x \in \overline{\{0\}} & \Leftrightarrow \operatorname{dist}_{\mathcal{P}}(x,\{0\})=\mathcal{P}(x, x) \\
& \Leftrightarrow \frac{3 x^{2}}{4}=\frac{x^{2}}{4} \\
& \Leftrightarrow x=0 \Leftrightarrow x \in\{0\} .
\end{aligned}
$$

Hence, $\{0\}$ is closed with respect to the partial symmetric $\mathcal{P}$. Next,

$$
\begin{aligned}
x \in \overline{\{0,1\}} & \Leftrightarrow \operatorname{dist}_{\mathcal{P}}(x,\{0,1\})=\mathcal{P}(x, x) \\
& \Leftrightarrow \min \left\{\frac{3 x^{2}}{2}, \frac{1}{2}|x-1|^{2}+\frac{1}{4}(\max \{x, 1\})^{2}\right\}=\frac{x^{2}}{4} \\
& \Leftrightarrow x \in\{0,1\} .
\end{aligned}
$$

Hence, $\{0,1\}$ is also closed with respect to the partial symmetric $\mathcal{P}$. Now, define $f: X \rightarrow \mathcal{C B}^{\mathcal{P}}(X)$ by:

$$
f 0=f 1=\{0\} \text { and } f 2=\{0,1\} .
$$

Notice that $f$ is continuous under the partial symmetric $\mathcal{P}$. Now, to show that the contractive condition (i) of Theorem 5 is satisfied, we distinguish the following cases:

Case 1. Let $x, y \in\{0,1\}$. Then,

$$
H_{\mathcal{P}}(f x, f y)=H_{\mathcal{P}}(0,0)=0,
$$


so that the contractive condition (i) satisfied.

Case 2. Let $x \in\{0,1\}$ and $y=2$. Then, with $k=\frac{1}{2}$, we have

$$
\begin{aligned}
H_{\mathcal{P}}(f x, f 2) & =H_{\mathcal{P}}(\{0\},\{0,1\}) \\
& =\max \left\{\delta_{\mathcal{P}}(\{0\},\{0,1\}), \delta_{\mathcal{P}}(\{0,1\},\{0\})\right\} \\
& =\max \left\{\operatorname{dist}_{\mathcal{P}}(0,\{0,1\}), \max \{\mathcal{P}(0,0), \mathcal{P}(1,0)\}\right\} \\
& =\frac{3}{4} \leq k H_{\mathcal{P}}(x, 2) .
\end{aligned}
$$

Case 3. Let $x=y=2$. Then, with $k=\frac{1}{4}$, we have

$$
\begin{aligned}
H_{\mathcal{P}}(f 2, f 2) & =H_{\mathcal{P}}(\{0,1\},\{0,1\}) \\
& =\delta_{\mathcal{P}}(\{0,1\},\{0,1\}) \\
& =\max \left\{\operatorname{dist}_{\mathcal{P}}(0,\{0,1\}), \operatorname{dist}_{\mathcal{P}}(1,\{0,1\})\right\} \\
& =\max \{0, \min \{\mathcal{P}(1,0), \mathcal{P}(1,1)\}\} \\
& =\frac{1}{4} \leq k H_{\mathcal{P}}(2,2)
\end{aligned}
$$

Hence, the contractive condition (i) of Theorem 5 is satisfied for $k=\frac{1}{2}$.

By routine calculation, one can verify the other conditions of Theorem 5. Observe that $f$ has a unique fixed point (namely, $x=0$ ).

\section{Conclusions}

First, we enlarged the class of symmetric spaces to the class of partial symmetric spaces, wherein we proved several results which included analogues of the Banach contraction principle, the Kannan-Ćirić fixed theorem, and the Ćirić quasi-fixed point theorem, in such spaces. We also furnished some examples, exhibiting the utility of our newly established results. Furthermore, we used one of the our main results to examine the existence and uniqueness of a solution for a system of Fredholm integral equations. Moreover, we extended the idea of Hausdorff distance to partial symmetric spaces, and proved an analogue of Nadler's fixed point theorem and some related results.

Author Contributions: All authors contributed equally in writing this article. All authors read and approved the final manuscript.

Funding: This research is not funded by any external agency.

Conflicts of Interest: The authors declare no conflict of interest.

\section{References}

1. Banach, S. Sur les operations dans les ensembles abstraits et leur application aux equations integrals. Fund. Math. 1922, 3, 133-181. [CrossRef]

2. Matthews, S.G. Partial metric topology. Ann. N. Y. Acad. Sci. 1994, 728, 183-197. [CrossRef]

3. Amini-Harandi, A. Metric-like spaces, partial metric spaces and fixed points. Fixed Point Theory Appl. 2012, 2012, 204. [CrossRef]

4. Czerwik, S. Contraction mappings in b-metric spaces. Acta Math. Inf. Univ. Ostrav. 1993, 1, 5-11.

5. Branciari, A. A fixed point theorem of Banach-Caccioppoli type on a class of generalized metric spaces. Publ. Math. 2000, 57, 31-37.

6. Huang, L.G.; Zhang, X. Cone metric spaces and fixed point theorems of contractive mappings. J. Math. Anal. Appl. 2007, 332, 1468-1476. [CrossRef]

7. Imdad, M.; Asim, M.; Gubran, R. Common fixed point theorems for g-Generalized contractive mappings in b-metric spaces. Indian J. Math. 2018, 60, 85-105. 
8. Ciric, L.B. A generalization of Banach's contraction principle. Proc. Am. Math. Soc. 1974, 45, $267-273$. [CrossRef]

9. Mustafa, Z.; Roshan, J.R.; Parvaneh, V.; Kadelburg, Z. Some common fixed point results in ordered partial b-metric spaces. J. Inequal. Appl. 2013, 2013, 562. [CrossRef]

10. Imdad, M.; Ali, J. Common fixed point theorems in symmetric spaces employing a new implicit function and common property (EA). Bull. Belg. Math. Soc. Simon Stev. 2009, 16, 421-433.

11. Jleli, M.; Samet, B. A generalized metric space and related fixed point theorems. Fixed Point Theory Appl. 2015, 2015, 61. [CrossRef]

12. Wilson, W.A. On semi-metric spaces. Am. J. Math. 1931, 53, 361-373. [CrossRef]

13. Nadler, S.B. Multivalued contraction mappings. Pac. J. Math. 1969, 30, 475-488. [CrossRef]

14. Villa-Morales, J. Subordinate Semimetric Spaces and Fixed Point Theorems. J. Math. 2018, 2018, 7856594. [CrossRef]

(C) 2019 by the authors. Licensee MDPI, Basel, Switzerland. This article is an open access article distributed under the terms and conditions of the Creative Commons Attribution (CC BY) license (http://creativecommons.org/licenses/by/4.0/). 